\title{
Primer reporte de Entoloma hochstetteri (Entolomataceae, Agaricales, Basidiomycota) para el departamento de Santander, Colombia
}

\section{First report of Entoloma hochstetteri (Entolomataceae, Agaricales, Basidiomycota) for the department of Santander, Colombia}

\author{
César Augusto Pinzón-Osorioํㅜ, Jonás Pinzón-Osorio²
}

\begin{abstract}
Resumen
Resultado: Se presenta una nueva localidad para Entoloma hochstetteri, por lo que se amplía su distribución geográfica en Colombia. Metodología: Los basidiocarpos se recolectaron en bordes de camino de herradura y en bosque muy húmedo subtropical ubicado en el corregimiento de Olival, municipio de Suaita, departamento de Santander, Colombia. Se realizaron tres expediciones entre los años 2013 y 2014, únicamente en el mes de julio. Todos los basidiocarpos se fotografiaron y describieron tanto macroscópica como microscópicamente siguiendo el tratamiento recomendado por autoridades en el campo micológico. Se da información sobre distribución y sustrato de crecimiento de la especie. Conclusiones: Los basidiomas se observaron en los 3 rastreos realizados en áreas de alto impacto agropecuario, lo que permitiría afirmar que esta especie se adapta a ambientes relativamente homogéneos. De acuerdo con las condiciones climáticas de la zona, se considera que el desarrollo de esta especie depende en gran medida de la humedad, característica de la época de colecta. Se espera encontrarla en otras regiones del país.
\end{abstract}

Palabras clave: Ampliación de distribución, Colombia, Entoloma hochstetteri, Micología, Nuevo registro, Santander.

\begin{abstract}
Results: A new location of the the Entoloma hochstetteri is reported, so that its geographical distribution spreads out in Colombia. Methodology: The basidiocarps were collected in the edges of bridle paths and in very humid subtropical forest located in the township of Olival, municipality of Suaita, department of Santander, Colombia. Three expeditions were carried out between 2013 and 2014, only in the month of July. All the basidiocarps were photographed and described macroscopically and microscopically following the recommended protocol by the authorities in the mycological field. Information on the distribution and growth substrate of the species is provided. Conclusion: The basidiocarps were observed in the three scan performed in areas of high agricultural impact, which would allow to state that this species adapts to relatively homogeneous conditions. According to the climatic conditions of the area, it is considered that the development of this species largely depends on the moisture, which is common during the collecting period. It is expected to find it in other regions of the country.
\end{abstract}

Keywords: Colombia, Expansion of distribution, Entoloma hochstetteri, Mycology, New record, Santander.

\section{Introducción}

De acuerdo con Raj et al. (2014) el género Entoloma s.l. es uno de los grupos más grandes del orden Agaricales Underw, con 1500 especies reportadas en todo el mundo (Kirk et al. 2008). Para Colombia, según Vasco-Palacios y Franco-Molano (2013) la familia Entolomataceae Kotl. \& Pouzar está representada por siete géneros distribuidos en trece especies, de las cuales cuatro corresponden al género Entoloma: E.

1 Programa de Licenciatura en Biología, Herbario, Departamento de Biología, Universidad Pedagógica Nacional, Sede Bogotá. Colombia. e-mail: cesar.fungi20@gmail.com

2 Programa de Ciencias Biológicas, Departamento de Ciencias Exactas y Naturales, Universidad de Buenos Aires, Buenos Aires, Argentina. e-mail: ad10jpin@uwcad.it

Fecha recepción: Agosto 11, $2014 \quad$ Fecha aprobación: Febrero 20, $2016 \quad$ Editor Asociado: Torres-Torres M 
clitocyboides E. Horak \& Singer, E. ferrugineogranulatum (Singer) E. Horak, E. lyophylliforme (Singer) E. Horak y E. hochstetteri (Reichardt) G. Stev.

Entoloma hochstetteri (Reichardt) G. Stev. también conocida como E. virescens sensu Horak., fue registrada por primera vez por el micólogo austríaco Reichardt (1866), al conocer una ilustración realizada por un coleccionista, quien encontró este hongo en bosques nativos de Nueva Zelanda. La ilustración fue descrita inicialmente como Hygrophorus hochstetteri (Reichardt).

Esta seta se caracteriza por presentar colores que van del azul oscuro a azul verdoso con tintes grisáceos (Alves y Coelho 2012). Puede variar a azul negruzco o azul amarillento con manchas oscuras por contacto, maduración o corte. El píleo es por lo general convexo a levemente cónico (Horak 1976). Microscópicamente las esporas presentan 4 ángulos, siendo cuboidales (Franco-Molano et al. 2005, Alves y Coelho 2012), de coloración rosa a marrón rosáceo cuando se encuentran en masa (Wright y Alberto, 2002). Los queilocistidios son cilíndricos a claviformes (Horak 1976).

En Colombia, la especie ha sido reportada únicamente para el departamento de Caquetá por
Franco-Molano et al. (2005) y Vasco-Palacios y Franco-Molano (2013). En esta ocasión, se presenta información micológica que amplía la distribución geográfica de E. hochstetteri para el departamento de Santander, Colombia.

\section{Métodos}

Área de estudio. La zona de estudio se localiza en el corregimiento de Olival, ubicado en las coordenadas $6^{\circ} 09^{\prime} 11.82^{\prime \prime} \mathrm{N}$ y $73^{\circ} 20^{\prime} 26^{\prime \prime} \mathrm{O}$, a $1700 \mathrm{msnm}$, municipio de Suaita, departamento del Santander (Figura 1). Se muestreó en la Finca El Rodeo, Monte del Águila y San Antonio.

La zona se caracteriza por presentar una temperatura mínima de $17.5^{\circ} \mathrm{C}$ y máxima de $24^{\circ} \mathrm{C}$, con precipitación promedio anual de $2850 \mathrm{~mm}^{3}$. De acuerdo con la clasificación de zonas de vidas de Holdridge (1971), en el área muestreada se presenta un bosque muy húmedo subtropical (bmh-ST), sometido a actividades de ganadería extensiva, cultivo de caña (Saccharum officinarum L.), yuca (Manihot esculenta Crantz.) y plátano (Musa paradisiaca L.).

Recolecta y determinación. Se llevó a cabo un muestreo tipo oportunístico, durante 9 días, en 4

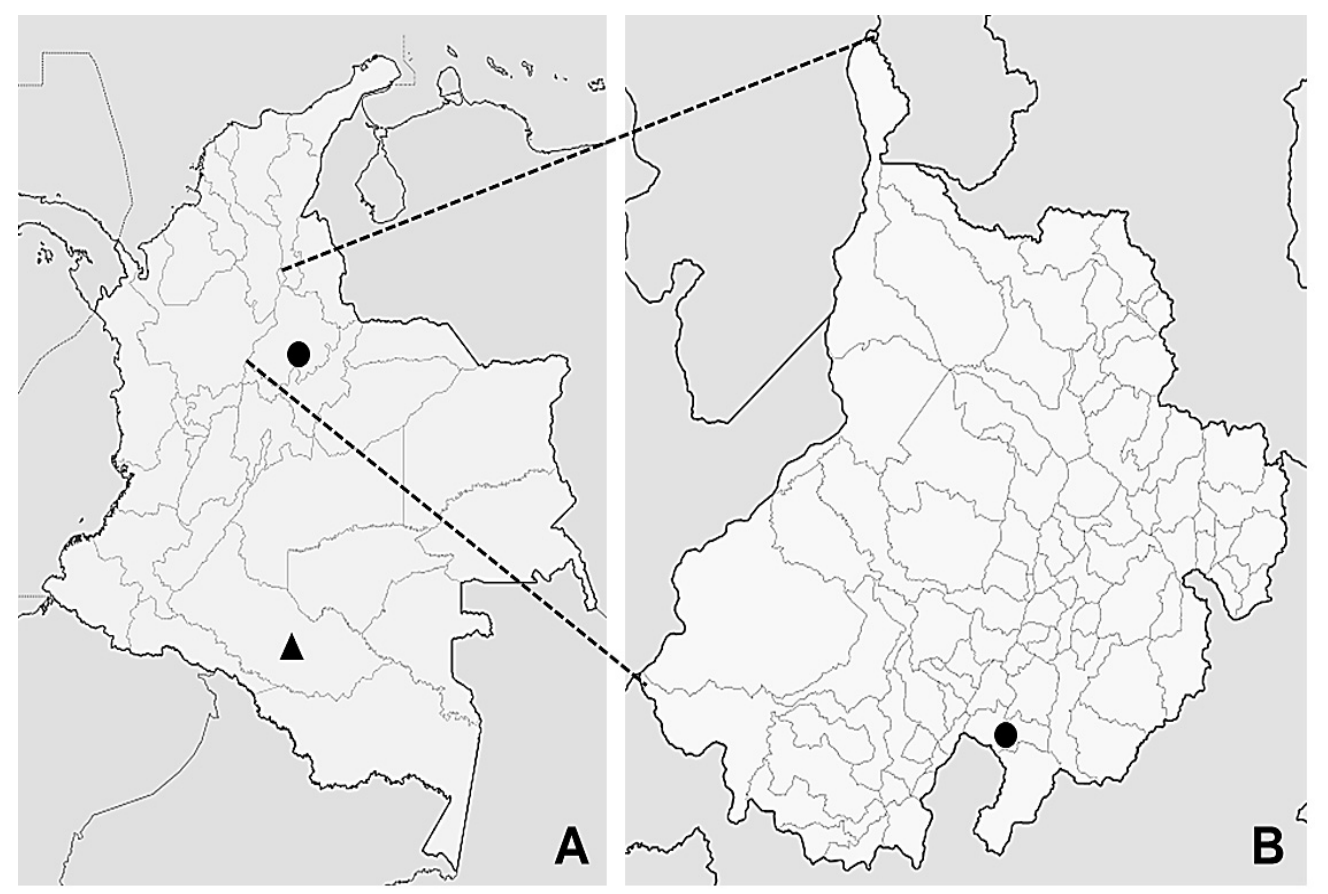

Figura 1. Distribución de Entoloma hochstetteri para Colombia. A. Registro previo en el departamento del Caquetá (A ) (Franco-Molano et al. 2005). B. Nueva zona de registro, departamento de Santander (•).

Tomado y modificado de la Alcaldía de Suaita, Santander http://www.suaita-santander.gov.co/mapas_municipio.shtml 

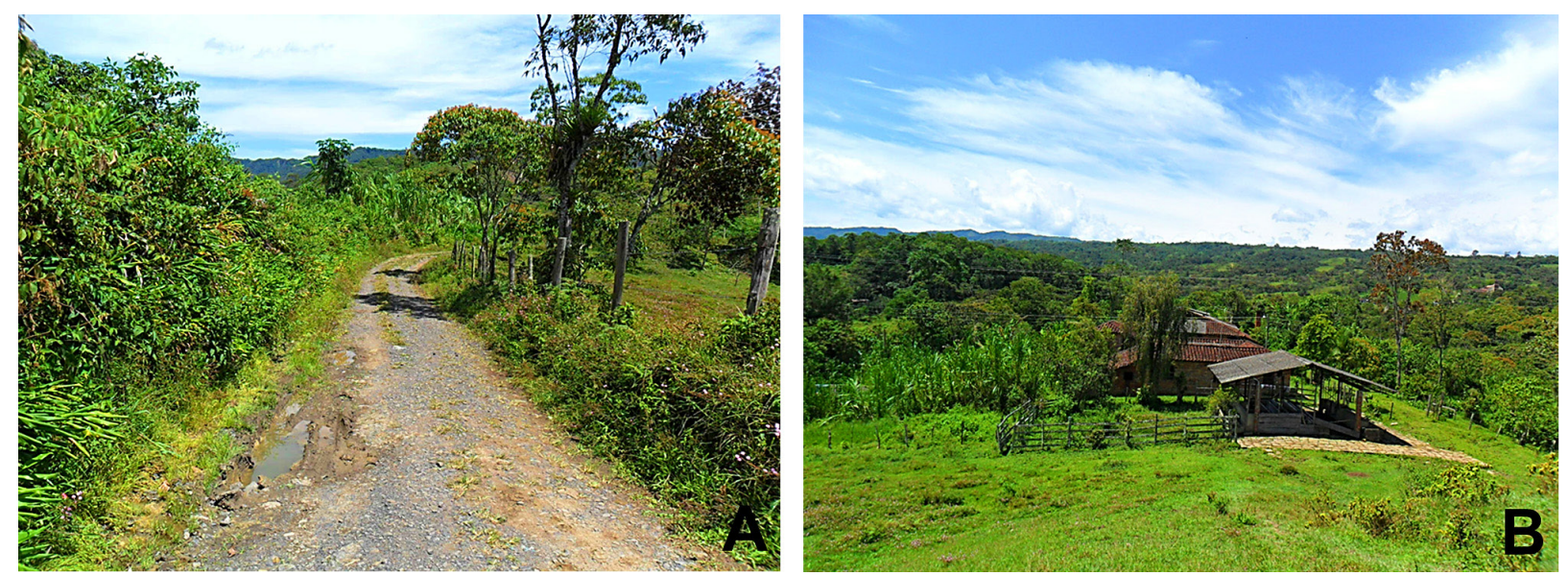

Figura 2. Áreas de recolecta. A. Camino de herradura. B. Finca El Rodeo con bosque muy húmedo subtropical.

hectáreas del ecosistema y en un camino de herradura que convergía con este (Figura 2).

Los basidiomas se fotografiaron, recolectaron y describieron macroscópicamente siguiendo las recomendaciones de Largent et al. (1986) y Franco-Molano et al. (2005). Luego se deshidrataron y depositaron en el Herbario de la Universidad Pedagógica Nacional, Sede Bogotá. Para la observación microscópica se realizaron cortes a mano alzada de los basidiomas, que se montaron en $\mathrm{KOH}$ al 5\% y rojo Congo. Se utilizó el reactivo de Melzer como prueba bioquímica para determinar la presencia de almidón en las basidiosporas. Las estructuras fúngicas se observaron en microscopio con sistema digital DMS 653 y se midieron con el software Piximétre. El material estudiado se identificó por comparación macroscópica y microscópica con lo reportado por Horak (1976), Franco-Molano et al. (2005), Largent y Abell-Davis (2011) y Alves y Coelho (2012). Los colectores se indican con iniciales: C. Pinzón (CP), J. Pinzón (JP).

\section{Resultados}

Entoloma hochstetteri (Reichardt) G. Stev., Kew Bull. 16(2): 233 (1962)

Píleo: 0.8-3.0 cm de diámetro, cónico, papilado cuando joven, convexo-plano al madurar, margen incompleto o discontinuo cuando madura, superficie lisa a fibrilosa, húmeda, brillante, líneas longitudinales del centro al margen, color azul claro a oscuro, algunas zonas con gris verdoso-amarillento con tintes azules. Contexto: hasta $0.1 \mathrm{~cm}$ de grosor, blanco, azul claro a azul oscuro. Olor: no registrado. Sabor: fúngico, algo ácido. Lamelas: hasta $0.15 \mathrm{~cm}$ de grosor, anexas, algunas libres, cercanas, gruesas, concoloro con el contexto, con polvillo rosado en las aristas. Lamélulas: 2 longitudes diferentes. Estípite: 4.0$10.7 \mathrm{~cm}$ de longitud por $0.2-0.6 \mathrm{~cm}$ de grosor, central a excéntrico, cilíndrico, levemente ensanchado en la base cuando madura, superficie lisa o fibrilosa, muy frágil, color azul a blanco plateado con tintes crema hacia la base (Figura 3 A-B).

Esporada: color rosado claro. Basidiosporas: 9.9-13 x 9.1-11.9 $\mu \mathrm{m}$, cuboidales, paredes relativamente delgadas, amarillentas en $\mathrm{KOH}$ (Figura 3 F), inamiloides en reactivo de Melzer. Basidios: 29.4-56.6 x 10-13.7 $\mu \mathrm{m}$, claviformes, con 2, 3 o 4 esterigmas, pared delgada, hialinos (Figura 3 C-D-E), algunos fibulados en la base. Pileipellis: un cutis, con hifas de hasta $13 \mu \mathrm{m}$ de ancho, fibuladas, con algunos elementos terminales, algunas con pigmento intracelular granular. Trama lamelar: paralela (Figura 3G). Pseudocistidios: 4-10.4 $\mu \mathrm{m}$ de diámetro, sinuosos, ligeramente estrechos en ápice, hialinos, de pared delgada. Queilocistidios: 24-50 x 10.2-15.5 $\mu \mathrm{m}$, cilíndricos a claviformes, hialinos o con contenido vacuolar de color café.

Hábito y hábitat: solitario a gregario, sobre tierra húmeda, asociado con musgos y helechos.

Uso tradicional o práctico: no registrados.

Colecciones examinadas: (2 julio-2013) (JP) $6^{\circ}$ 09' 09,04" N y 73²0' 25,34" O. Finca El Rodeo, 1500 msnm (20 julio-2013) (CP) 6 ${ }^{\circ} 09^{\prime} 09,12$ " N y $73^{\circ} 20^{\prime} 25,27^{\prime \prime}$ O. Monte del Águila, $1680 \mathrm{msnm}$. (20 julio-2013) (JP) 6 69'09,04" N y 73²0'25,34” O Finca El Rodeo, 1500 msnm (5 julio-2014) (CP) $6^{\circ}$ 


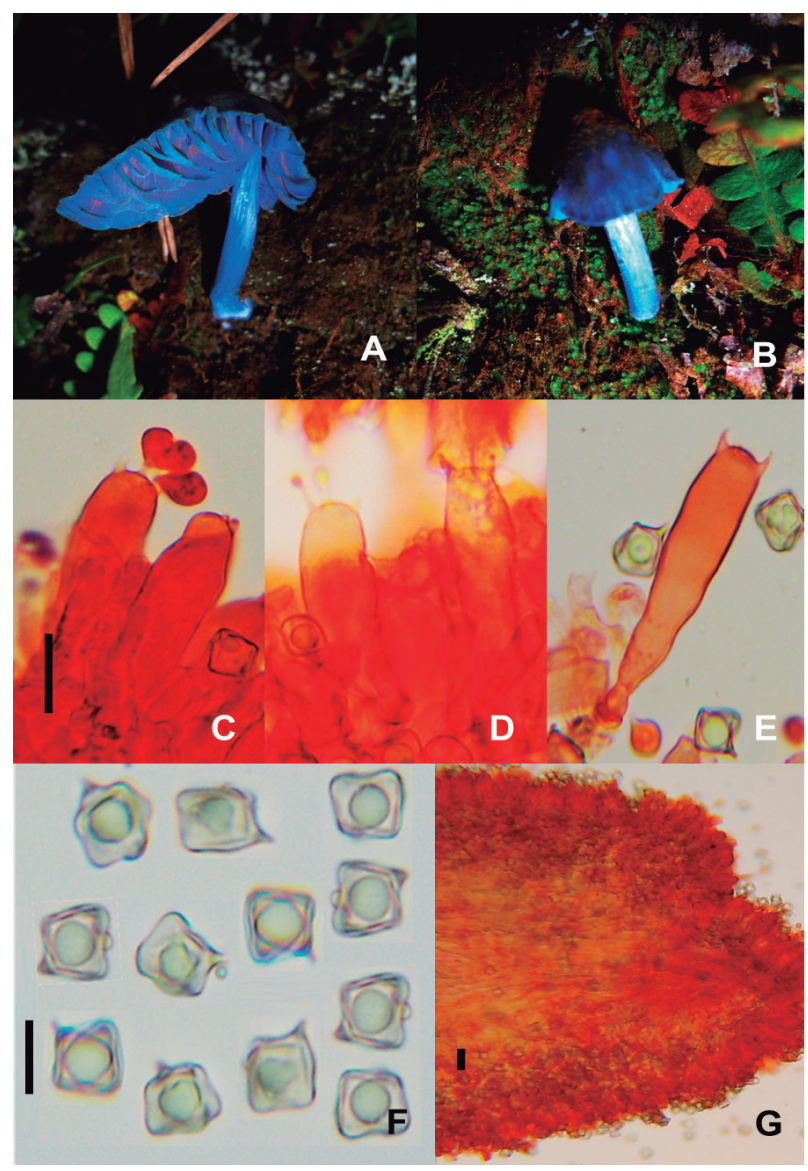

Figura 3. Registro fotográfico macroscópico y microscópico de Entoloma hochstetteri. A. Macromiceto joven-maduro. B. Basidiocarpo joven. C. Basidios 4-sterigmatado (4) esporas. D. 3-sterigmatado (3) esporas. E. 2-sterigmatados (2) esporas. F. Basidiosporas. G. Trama lamelar. Líneas $10 \mu \mathrm{m}$.

Fotografías: César Pinzón

09'09,12" N y 73²0' 25,27" O Monte del Águila, 1580 msnm (5 julio-2014) (CP) 6 $09^{\prime} 11,84^{\prime \prime} \mathrm{N} \mathrm{y}$ $73^{\circ} 20^{\prime} 26,04$ ' O. San Antonio. $1500 \mathrm{msnm}$.

Distribución: esta especie presenta amplia distribución. Se considera que la región que abarca a Malasia y Nueva Zelanda es el epicentro de dispersión (Horak 1976). Pegler (1986) registró la especie en Tailandia y Zambia, Horak (1976) lo hizo en Nueva Zelanda, Japón, Isla de Bonin, Malasia, Madagascar, Papua Nueva Guinea y Sri Lanka. El mismo autor demostró su existencia en el trópico y subtrópico. En América del Sur E. hochstetteri se reportó en Brasil (Pegler 1997, Largent y Abell-Davis 2011, Alves y Coelho 2012).

En Colombia solo se reportaba en el departamento de Caquetá (Franco-Molano et al. 2005, Vasco-Pala- cios y Franco-Molano 2013). El presente manuscrito amplía su distribución al norte del país, en el departamento de Santander, municipio de Suaita, Olival, en bosque muy húmedo subtropical fragmentado. El área de muestreo presenta un entorno altamente impactado por actividad de tipo agrícola y ganadería, por lo que este es el primer reporte de la especie en ecosistemas de estas características.

Observaciones: La descripción de las estructuras concuerda muy bien con la realizada por Alves y Coelho (2012), sin embargo, las medidas macroscópicas y microscópicas difieren de otros registros. El estípite no superó los $10.7 \mathrm{~cm}$ de longitud, $1.3 \mathrm{~cm}$ menos que el reportado por Franco-Molano et al. (2005). El tamaño del píleo no alcanzó los $3.5 \mathrm{~cm}$ de diámetro señalados por los mismos autores.

Microscópicamente, la descripción realizada por Franco-Molano et al. (2005) registran basidiosporas con dimensiones que oscilan entre 9-11 x 8-10 $\mu \mathrm{m}$. Horak (1976) reporta medidas entre 11-15 x 11-14 $\mu \mathrm{m}$. Pegler (1997), con un estudio en Suramérica evidenció tamaños de 10-14 x 9-11 $\mu \mathrm{m}$. Para el presente reporte, las esporas de E. hochstetteri son de 9.9-13 x 9.1-11.9 $\mu \mathrm{m}$, lo que demuestra que los especímenes recolectados en Santander, Colombia difieren de lo mencionado por Horak (1976), Pegler (1997) y Franco-Molano et al. (2005). Las medidas coinciden mejor con las reportadas en Brasil por Alves y Coelho (2012), 9.75-13.75 x 9.75-12.5 $\mu \mathrm{m}$.

Los basidios no alcanzaron el diámetro reportado por Horak (1976) y Alves y Coelho (2012), 10-20 $\mu \mathrm{m}$ y $14-18 \mu \mathrm{m}$, respectivamente. Las dimensiones, sin embargo, no exceden el límite inferior de los mencionados por Horak (1976). El diámetro de los pseudocistidios y queilocistidios son similares a las descritas por Alves y Coelho, (2012), 6-9.2 $\mu \mathrm{m}$ de diámetro y 28-55.5 x 11-16.75 $\mu \mathrm{m}$, respectivamente.

El hábito de crecimiento reportado para el departamento de Santander difiere con el registrado en Queensland, Australia (Largent y Abell-Davis 2011) y en Caquetá, Colombia (Franco-Molano et al. 2005), citados como solitarios. El nuevo reporte presentó un hábito tipo solitario a gregario, agrupándose hasta un máximo de 3 carpoforos.

\section{Conclusión}

Basado en la similitud morfológica tanto macros- 
cópica como microscópica que mostraron los especímenes con lo reportado en la literatura especializada, estos coinciden con E. hochstetteri. Por tanto, este trabajo amplía la distribución de la especie al norte del país. Los basidiomas fueron observados en los 3 rastreos realizados en áreas de alto impacto agropecuario, lo que permitiría afirmar que esta especie se adapta a condiciones relativamente homogéneas, teniendo en cuenta el tratamiento agrícola al que es expuesto el suelo. Esta especie presenta una amplia distribución y puede desarrollarse en diferentes hábitats. De acuerdo con la comunicación personal con los pobladores de la zona, esta especie fructifica en períodos de alta humedad, característica común del mes de julio, aspecto que concuerda con lo informado en otras áreas de Suramérica como Brasil, donde se han registrado en regiones con clima templado a húmedo. Sin embargo, se requiere extender los períodos de muestreo durante los diferentes meses del año y complementar con estudios ecológicos y fenológicos para evaluar el comportamiento de fructificación de esta especie para la zona estudiada.

\section{Agradecimientos}

Los autores agradecen la colaboración de Pablo Osorio Niño, propietario de las tres áreas rastreadas, quien amablemente permitió la realización del presente estudio en sus predios. A la Línea de Investigación Enseñanza y Aprendizaje de la Botánica de la Universidad Pedagógica Nacional. Al especialista Juventino García Alvarado de la Universidad Veracruzana de México por la revisión y los comentarios críticos al manuscrito. Este artículo está dedicado a Leonel Gil.

\section{Literatura citada}

Alves MH, Coelho C. 2012. Entoloma virescens (Sacc.) E. Horak ex Courtec. 1986 (Agaricales: Entolomataceae): The first record for the Caatinga biome, Ceará, Brazil. Check List. 8 (3): 577-80.

Franco-Molano AE, Vasco AM, López CA, Boekhout T. 2005. Macrohongos de la región del medio Caquetá-Colombia. Guía de Campo. Grupo de Taxonomía y Ecología de Hongos. Medellín: Universidad de Antioquia; $211 \mathrm{pp}$.

Holdridge LR, Grenke W, Hatheway WH, Liang T, Tosi JA. 1971. Forest environments in tropical life zones: A pilot study. Oxford: Pergamon Press; 747 pp.

Horak E. 1976. On cuboid-spored species of Entoloma (Agaricales). Sydowia. 28 (1-6): 171-237.

Kirk PM, Cannon PF, Minter DW, Stalpers JA. 2008. Dictionary of the fungi. 10th ed. Wallingford: CABI Publishing; 640 pp.

Largent D, Abell-Davis SE. 2011. Observations on Inocephalus virescens comb. nov. and Alboleptonia stylophora from northeastern Queensland. [Revista en línea]. [Acceso 25 de junio del 2014]. Mycotaxon. 116: 231-45. Disponible en: http://www.ingentaconnect.com/content/mtax/ $\mathrm{mt} / 2011 / 00000116 / 00000001 /$ art00027? crawler=true

Largent D, Johnson D, Watling R. 1986. How to identify mushrooms to genus III: microscopic features. Eureka: Mad River Press; 273 pp.

Pegler DN. 1977. A preliminary Agaric flora of East Africa. Kew Bull Additional Ser. 6: 620.

Pegler DN. 1986. Agaric flora of Sri Lanka. Kew Bull Additional Ser. 12: 519.

Raj KNA, Latha KPD, Kumar TKA, Manimohan P. 2014. A new species of Entoloma from India. Mycoscience. 55 (5): 400-4.

Reichardt HW. 1866. Diagnosen der neuen Arten von Pilzen, welche die Novara-Expedition mitbrachte. Verh Zool Biot Ges Wien. 16: 373-6.

Vasco-Palacios AM, Franco-Molano AE. 2013. Diversity of Colombian macrofungi. (Ascomycota-Basidiomycota). [Acceso 27 marzo del 2014]. [Revista en línea]. Mycotaxon. 121: 1-58. Disponible en: https://mycotaxon.com/ resources/checklists/VascoPalacios-v121-checklist.pdf

Wright J, Alberto E. 2002. Hongos: Guía de la región Pampeana. I Hongos con Laminillas. Buenos Aires: Ed. LOLA; 279 pp. 